\title{
Universitätslehrgang Master of Public Health: Prävention und Gesundheitsförderung
}

Der Master-Lehrgang der beiden Wiener Universitäten bietet in Form eines viersemestrigen, berufsbegleitenden postgradualen Studiums die Möglichkeit, auf dem Gebiet der integrativen Prävention und Lebensstilmedizin die entsprechenden Kompetenzen und Fähigkeiten zu erwerben, um sie im intrawie extramuralen Bereich zielgruppenorientiert im Sinne der Öffentlichen Gesundheit (Public Health) anwenden und evaluieren zu können. Damit verbunden sind der Erwerb von umfassenden Wissensgrundlagen und fachlichen Qualifikationen für Führungsaufgaben und Leitungsfunktionen im Gesundheitswesen mit Schwerpunkt Prävention und Gesundheitsförderung.

Das interdisziplinäre Curriculum des Master-Studienganges in Public Health setzt den Schwerpunkt in der Aus- bildung für Lebensstilmedizin mit besonderer Berücksichtigung der Leistungs-, Ernährungs- und Sozialmedizin, Epidemiologie und Gesundheitsmanagement mit besonderer Berücksichtigung hinsichtlich Kompetenzen zur nachhaltigen Umsetzung des erworbenen Wissens in der Praxis der Gesundheitsförderung und Prävention.

\section{Zielgruppe/Voraussetzungen} für die Teilnahme:

\section{Postgraduell}

Voraussetzung für die Teilnahme an dem Universitätslehrgang sind der Abschluss einer in Österreich anerkannten Ausbildung zum Dr. med. univ., Dr. med. dent., Dr. med. scient., oder eine gleichgestellte ausländische

\section{Universitätslehrgang Master of Public Health:} Prävention und Gesundheitsförderung

Abschluss: Akademischer Titel Master of Public Health

Unterrichtssprache: Deutsch

Dauer: 4 Semester (berufsbegleitend), 120 ECTS

Beginn des nächsten Lehrgangs: 9. Oktober 2009

Anmeldung: ab sofort möglich

Kosten für den gesamten Lehrgang: $€ 11.500$,-

Informationen und Anmeldung:

Universitätslehrgang Master of Public Health Vienna

Zentrum für Sportwissenschaft und Universitätssport der Universität Wien

c/o Sekretariat Regina Kreiter

Auf der Schmelz $6 a$

A-1150 Wien

Tel.: +43-676-7359759

Fax: +43-1-4277-59199

E-Mail: public.health@univie.ac.at

Homepage: www.mph-vienna.at
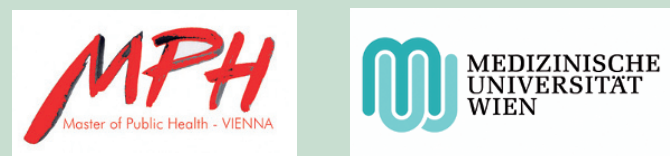

Graduierung beziehungsweise der Abschluss eines facheinschlägigen naturwissenschaftlichen, wirtschaftswissenschaftlichen, rechtswissenschaftlichen oder geistes- und sozialwissenschaftlichen Diplomstudiums oder Abschluss eines Studiums an einer anerkannten in- oder ausländischen postsekundären Bildungseinrichtung (z. B. Fachhochschule), das den oben genannten Diplomstudien gleichwertig ist. Die Gleichwertigkeit ist vom Rektor im Rahmen des Zulassungsverfahrens festzustellen. Von allen Teilnehmern wird ein nachweisbares Interesse zum Thema Prävention und Gesundheitsförderung verlangt, welches auf dem Anmeldeformular und/oder in einem Aufnahmegespräch mit der Lehrgangsleitung festgestellt wird.

\section{Zulassungsvoraussetzung für} Nichtakadmiker/innen mit erfolgreicher Studienberechtigungsprüfung (Matura)

mindestens 5 Jahre Berufserfahrung in den Bereichen Public Health, Krankenpflege, medizinisch-technische Berufe, soziale Berufe und entsprechende fachverwandte Berufe odermindestens 3 Jahre Praxis in verantwortlicher Führungsposition im Gesundheitsbereich oder Nachweis von mindestens 120 ETCS-Credits eines in Österreich anerkannten, facheinschlägigen medizinischen, naturwissenschaftlichen, wirtschaftswissenschaftlichen, rechtswissenschaftlichen oder geistes- und sozialwissenschaftlichen Diplom- oder Bakkalaureatstudiums und mindestens 2 Jahre Berufserfahrung in den Bereichen Public Health, Krankenpflege, medizinisch-technische Berufe, soziale Berufe und entsprechende fachverwandte Berufe 